Version non éditée, voir : Francesca Artioli. Les politiques urbaines d'Italie comme miroir pour la

France : différences, réformes, circulations. Banlieues vues d'ailleurs, CNRS Éditions, pp.159 - 174,

2016, Les Essentiels d'Hermès

http://www.cnrseditions.fr/communication/7239-banlieues-vues-d-ailleurs.htm

\title{
Les politiques urbaines d'Italie comme miroir pour la France : différences, réformes, circulations
}

En mettant en lumière les acteurs et les instruments des politiques pour les villes italiennes et leurs périphéries, cet article apporte un éclairage sur les modes de gouvernance en France. Mettre les périphéries et les politiques françaises au miroir de celles italiennes a ici une double signification. D'une part, il s'agit de raconter les changements des villes d'outre-alpes, les politiques qui y sont mises en œuvre et les récits que les acteurs politiques et académiques développent sur le cas italien en comparaison avec la France et l'Europe. D'autre part, il s'agit d'offrir au lecteur français un détour par autre système urbain et national pour apporter une nouvelle perspective. Pour ce qui nous intéresse ici, à savoir les politiques et les modes de gouvernance des villes et des quartiers, cette mise au miroir est d'autant plus intéressante que la France et l'Italie sont deux pays similaires sur le plan des institutions. Ce sont deux États unitaires et historiquement centralisés, avec des collectivités locales ayant des ressources faibles et dépendantes des ressources du centre national. Les deux pays ont entamé un processus de réorganisation de leurs administrations territoriales depuis une trentaine d'années, marqués par un transfert de compétences vers les échelons infranationaux (avec une régionalisation plus poussée en Italie). Leurs dynamiques urbaines sont néanmoins marquées par de différences structurelles qui soulèvent la question des effets des circulations des modèles et des stratégies de réforme politique.

\section{Un pays de villes aux politiques urbaines tardives}

Dans l'histoire de l'Italie républicaine, la ville ne devient un objet explicite d'intervention par les autorités publiques qu'à la fin des années 1980. Cette absence est d'autant plus saisissante au égard du caractère urbain du pays : l'Italie dispose d'un tissu urbain à la fois dense et diffus qui ne connaît pas la macrocéphalie de la ville capitale que caractérise Paris et la France ou Londres et le RoyaumeUni. Comme cela est le cas dans la plupart des pays d'Europe occidentale qui connaissent dans ces années un boom économique, les problèmes des périphéries sont à cette époque ceux liés au souséquipement et à une demande de logements et de services importante par les nouveaux habitants de villes qui sont, en Italie, issus de la croissance démographique, ainsi que du double mouvement de la campagne vers la ville et du Mezzogiorno vers le Septentrion.

Si l'on prend la création d'une administration dédiée comme un indicateur pour saisir si et comment une question est traitée par les pouvoirs publics, on observe que le premier ministère pour les villes est crée en Italie en 1987. De l'après-guerre jusqu'à la fin des années 1980, donc, il n'y a pas de politiques urbaines nationales, c'est-à-dire des politiques transversales qui prennent pour cible explicite les villes ou les quartiers comme territoires particuliers auxquels on associe un ensemble de problématiques particulières (Allulli, 2010). Pour autant, ceci ne signifie pas qu'il y a absence totale d'intervention publique sur les villes et les quartiers en Italie. Elle prend ici deux formes principales. En premier lieu, elle repose sur les gouvernements municipaux. Dans un contexte marqué par un État centralisé et à l'architecture institutionnelle d'inspiration napoléonienne, donc similaire au cas français, les gouvernements urbains ont des compétences et des capacités financières limitées pour d'élaboration 
et mise en œuvre de politiques publiques. Ils disposent en revanche de canaux politiques (fournis notamment par les partis politiques) pour représenter au centre national les intérêts territoriaux et obtenir des ressources (Tarrow, Katzenstein, \& Graziano, 1978). De l'articulation entre liens verticaux des acteurs politiques locaux et capacités administratives locales naissent des expériences novatrices qui font des gouvernements urbains de fournisseurs importants de services publics relevant de l'État providence. En deuxième lieu, certaines politiques nationales sectorielles fonctionnent, de facto, comme des politiques urbaines. C'est notamment le cas de la politique de construction de logements abordables après la deuxième guerre mondiale. Dans les années 1950 jusqu'à la fin des années 1970 (moment du dernier financement conséquent dans le logement public), l'État central promeut des programmes de construction de quartiers résidentiels, dans les plans Vanoni et celui Fanfani pour le logement, qui fonctionnent à travers l'articulation entre financement national et une réalisation de logements basée sur des accords entre villes et sociétés coopératives. Dans ces décennies, la gestion du logement s'inscrit dans la logique des politiques nationales de la planification et de la modernisation économique, que l'on peut lire comme des politiques de transformation de la société élaborées dans les instances décisionnelles nationales élues, mais appuyées sur la concertation entre parties sociales.

\section{Innovation et rénovation urbaine entre les villes et les modèles européens, le tournant des années 1990}

Si en 1987 est crée le Ministère pour les Aires Urbaines - qui peut être considéré comme la première institutionnalisation de la ville comme objet, territorial et non sectoriel, dintervention nationale - la construction d'un nouvel ensemble de politiques urbaines dans les années 1990 est le fait d'autres acteurs et dynamiques. En effet, dans leurs approches, contenus et modes de gouvernance, les nouvelles politiques sont marquées par les programmes urbains de l'Union européenne, par la mobilisation de modèles issus des politiques françaises et britanniques, ainsi que par les changements des institutions locales du pays (notamment le transfert de compétences aux régions et aux autres échelons de gouvernements infranationaux, ainsi que l'introduction de l'élection du maire au suffrage direct). Sans rentrer dans le détail de l'ensemble des politiques publiques, il importe de souligner l'émergence, entre autres, de nouveaux programmes de rénovation urbaine qui ciblent des quartiers paupérisés ou périphériques.

Un ensemble d'initiatives - dit de "programmes urbains complexes ${ }^{1}$ se donne l'objectif d'agir sur la ville à travers une «nouvelle» approche localisée et intersectorielle (Crosta, 1998 ; Bricocoli \& Cementeri, 2005 ; Vicari Haddock, 2005). Partant du constat des processus d'exclusion et de marginalisation sociaux que caractérisent certains quartiers (voir infra), les « contrats de quartier » sont un bon exemple du fonctionnement des programmes urbains complexes. Cette politique est lancée par le ministère des Travaux Publics en 1997 et s'inscrit dans un contexte de réformes marqué par la décentralisation et la contractualisation des politiques sociales. Les contrats de quartier sont finalisés à la «requalification urbanistique et sociale » des quartiers de logements sociaux de propriété publique marqués par une paupérisation et une dégradation du bâti. Ils font du quartier le lieu et l'objet de nouvelles intersections entre politiques sectorielles différentes, en premier l'urbanisme, les politiques sociales et la politique du logement. Dans le paysage des politiques italiennes de l'époque, un premier facteur de nouveauté réside dans le fait de prendre un territoire particulier - un quartier - comme objet

1 On peut citer notamment les programmes suivants: Programmi Integrati di Intervento (1992), Programmi Integrati di Intervento (1993), Programmi di Riqualificazione Urbana e Sviluppo Sostenibile del Territorio (1994), Contratti di Quartiere (1997). 
d'intervention, plutôt que de s'adresser à des catégories sociales spécifiques ou d'adopter une approche universaliste. Par ailleurs, ces programmes reposent sur l'idée du partenariat, à la fois entre des acteurs publics à différentes échelles et entre acteurs publics et privés, comme élément clé pour la définition des problèmes et l'élaboration des solutions. Enfin, c'est le principe de l'implication et de l'activation des groupes et des territoires bénéficiaires qu'oriente l'action. Si cette activation locale peut être une manière pour concevoir des projets qui soient adaptées aux spécificités de chaque contexte et " proches » des habitants, elle est également liée à une mise en compétition des territoires pour accéder aux ressources nécessaires au financements et à la mise en œuvre des projets, soient-elles nationales ou européennes .

D'où viennent ces politiques? D'une part, des facteurs que l'on peut définir d'endogènes au système politique italien sont à l'origine de cet essor de nouvelles interventions sur les périphéries. Sur le plan politique, le début des années 1990 est marqué par la disparition ou la transformation de principaux partis qui avaient gouverné le pays depuis l'après guerre. En même temps, la décennie est marquée par une vague de réformes institutionnelles qui assignent des responsabilités accrues aux gouvernements infranationaux et qui introduisent l'élection directe du maire (avant élus par le conseil). La délégation des responsabilités administratives de l'État vers les échelons régionaux, notamment, fait de ces nouvelles politiques une manière pour développer les capacités administratives des régions. Ces dernières utilisent leur responsabilité dans la gestion des fonds européens dans le domaine de la rénovation urbaine (programme Urban, voir infra) pour piloter l'action des gouvernements urbains, à travers l'introduction de critères auxquels est conditionné l'octroi de fonds. Par ailleurs, dans un contexte de crise et délégitimation de la politique nationale, les villes deviennent et sont vues comme les nouveaux lieux d'expérimentation et de ré-légitimation des capacités de la politique à traiter et résoudre les problèmes des territoires et des sociétés localisées, ce qui constitue une incitation à l'implication dans la rénovation urbaine (Lippi, 2011).

D'autre part, ces programmes ne se comprennent pas sans tenir compte de l'affirmation de paradigmes d'action publique au niveau européen et international. Les programmes de l'Union européenne pour les villes qui démarrent en 1994 on un effet direct. Le "programme d'initiative communautaire » URBAN fait l'objet en Italie d'un fort engouement et touche 16 villes dans sa première édition, puis une dizaine dans la deuxième. Plus généralement, il y a des processus mimétiques et d'apprentissage par l'Italie et vis-à-vis d'autres pays européens. En effet, tous les observateurs le soulignent, les politiques italiennes pour les périphéries s'appuient sur les expériences des politiques françaises et britanniques qui, une dizaine d'années avant, se sont données les quartiers et leurs problèmes comme objets privilégiés d'intervention. La Politique de la Ville française, notamment, a constitué un exemple direct pour l'institution en Italie des contrats de quartier, de politiques de zones franches urbaines et de pactes locaux pour la sécurité.

Cette ouverture à l'apprentissage et à l'activation de transferts de politiques publiques fournit de solutions à des problèmes qui sont identifiés comme étant «similaires » et légitime les politiques nationales à l'aune de «bonnes pratiques » étrangères. Néanmoins, l'existence de problèmes identiques des périphéries nécessitant des mêmes solutions est souvent remise en question, en raison des articulations entre marché du logement, pauvreté et ségrégation urbaine que caractérisent chaque pays et, notamment, différencient l'Italie de la France et des pays d'Europe du Nord (Bricocoli \& Cucca, 2014).

\section{Quelles politiques pour quels quartiers?}

Un certain nombre de changements structurels - désindustrialisation et changement des secteurs d'emplois, vieillissement, arrivée de populations d'origine étrangère - ont produit des trajectoires des quartiers périphériques des villes italiennes qui ont certainement des caractéristiques 
similaires à ceux d'autres pays Européens. Toutefois, de différences importantes existent tant pour ce qui relève des politiques et des problèmes de logement que pour ce qui est de la ségrégation urbaine. L'Italie comme de nombreux autres pays méditerranéens est caractérisé par le fait que les habitants sont, en vaste majorité, propriétaires de leur logement et la possession du logement de propriété est transversale à plusieurs classes sociales (Bricocoli \& Cucca, 2014). En 2008, la proportion de résidents dans un logement de propriété était de $81,5 \%$, avec une concentration des habitants en location vers les couches les plus défavorisées (CITTALIA, 2010). Dans ce cadre, des inégalités en matière de logement relèvent avant tout des temporalités inégales d'accession à la propriété, des lieux et des conditions d'achat et du poids financier que représentent l'achat et l'endettement pour les ménages.

Une deuxième caractéristique de l'Italie en comparaison avec la France est la très faible proportion de logement sociaux dans l'ensemble du parc : ils représentent $4 \%$ (CITTALIA, 2010). De plus, en Italie comme dans d'autres pays européens, des politiques de ventes aux occupants du parc social ont été mises en ouvre depuis une vingtaine d'années. Ajouté au fait que depuis trente ans, très peu de projets de construction de logements sociaux ont vu le jour, ceci a eu pour effet une réduction de longue durée du parc social disponible qui est passé de 900.000 logements en 1991 à 800.000 en 2007. En même temps, la construction a baissé de 34.000 logements à 1.900 en 2004. Par conséquent, si les zones périphériques marquées par les grands programmes de logement social réalisés dans les années 1970, sont celles qui concentrent le plus de précarité sociale, ces zones sont quantitativement inférieures à celles que l'on trouve en France. Ces programmes de logements connaissent aujourd'hui un état de délabrement qui se rajoute au caractère mono-fonctionnel de ces sites et un manque de ressources et services publics, y compris les transports en commun.

Enfin, par rapports à d'autres pays européens - tels que la France, le Royaume-Uni ou l'Allemagne - l'Italie a connu une immigration plus tardive et très rapide. Elle a été importante et concentrée pendant les deux dernières décennies: les étrangers en Italie sont 5,5 millions (dont environ $6 \%$ en situation irrégulière) $)^{2}$. Dans ce contexte de stock de logement social réduit et en décroissance, l'accès au logement social par la population immigrée est relativement rare. Le cas de Milan, par exemple, montre que la population immigrée trouve des possibilités de logement dans les quartiers les plus fragiles de la ville consolidée, où réside souvent une population âgée autochtone. Il s'agit de quartiers semi-centraux ou de périphérie intermédiaire qui datent d'avant la guerre et qui sont assez bien connectés au reste de la ville (Mingione, Borlini, \& Vitale, 2009).

Ces caractéristiques structurelles de l'Italie ont plusieurs conséquences. D'abord, elles aident à comprendre pour quelle raison les observateurs dressent le constat d'une différence entre les villes italiennes et celles françaises et anglaises en matière de ségrégation spatiale. Alors que la France ou le Royaume-Uni sont identifiés comme étant caractérisés par des formes ségrégation importantes, tant socio-économique qu'ethnique, l'Italie démure marquée par de niveaux de ségrégation spatiale moindre et un système basé sur la propriété. Néanmoins, ce manque de formes de ségrégation des immigrés ne doit pas amener à conclure à l'absence de conflits. La conflictualité dans les interactions multiethniques est importante, non seulement dans les grandes métropoles, mais aussi dans les plus petites villes et dans les districts productifs (Mingione et al., 2009).

Par ailleurs, les trajectoires d'urbanisation et du stock d'habitations en Italie expliquent le retour en force de la question du logement dans le pays, longtemps marginal dans les agendas politiques et médiatiques nationaux et urbains. La nouvelle demande de logement est liée aux processus conjoints d'augmentation du nombre de ménages et de la diminution du nombre de membres, des la demande liée aux migrations et au cycle d'augmentation forte des prix à l'achat et à la

2 Données de la Fondazione ISMU : http://www.ismu.org/2014/11/numeri-immigrazione/ 
location des décennies 1990 - 2000. La production et la fourniture de logements reposant sur le privé, il y a eu une augmentation du mal logement urbain et une nouvelle demande de logement abordable (CITTALIA, 2010).

Ces transformations rapides des villes et ces demandes trouvent aujourd'hui une réponse politique intermittente. Après quinze ans, les politiques contractuelles et basées sur le ciblage territorial s'essoufflent, dans les pratiques ainsi que dans les discours. La mise en évidence des effets que les choix régionaux et nationaux de politiques économiques et sociales ont sur la ville contribue à la critique de l'identification du local comme étant à la fois un problème et le point de départ de ses solutions. Les défaillances dans la mise en œuvre des programmes - avant tout la gestion des fonds européens - sont également soulignées. Enfin, la centralité de l'action municipale qui caractérise les politiques urbaines des décennies 1990 - 2000 est remise en cause. En effet, les relations villes/Etat en Italie fonctionnent aujourd'hui plus comme une distribution de la pénurie par l'État que comme un transfert de ressources. Ceci s'accompagne de formes de ré-centralisation non seulement financières mais également institutionnelles et discursives (Bolgherini, 2014 ; Dente, 2012). Les marges de manœuvre des villes sont aujourd'hui réduites et la pénurie de ressources financières pousse à favoriser l'urbanisation privée, les revenus tirés de la taxation de l'urbanisation permettant partiellement de couvrir les dépenses courantes des communes (Tosi \& Vitale, 2011)

\section{Références}

Allulli, M. (2010). Le politiche urbane in Italia. Tra adattamento e frammentazione. Roma : CITTALIA.

Bolgherini, S. (2014). Can Austerity Lead to Recentralisation? Italian Local Government during the Economic Crisis. South European Society and Politics, 19(2), 193-214. doi :10.1080/13608746.2014.895086

Bricocoli, M., \& Cementeri, L. (2005). Abitare: tra l'alloggio e la città. Quando le politiche entrano in casa. Dans Le nuove politiche sociali. Problemi dell'azione pubblica. Roma : Carocci.

Bricocoli, M., \& Cucca, R. (2014). Social mix and housing policy: Local effects of a misleading rhetoric. The case of Milan. Urban Studies, 0042098014560499. doi : $10.1177 / 0042098014560499$

CITTALIA. (2010). Comuni e la questione abitativa. Le nuove domande sociali, gli attori e gli strumenti operativi. Roma : (s.n.). Repéré à http://www.anci.it/Contenuti/Allegati/Questione\%20abitativa.pdf

Crosta, P. (1998). Politiche. Quale conoscenza per l'azione territoriale. Milano : Franco Angeli. Repéré à http://www.francoangeli.it/Ricerca/Scheda_libro.aspx?codiceISBN=9788846406019

Dente, B. (2012). The end of an era? The Monti government approach to central-local relations. LIEPP Working Papers, (6), 1-21.

Lippi, A. (2011). Evaluating the 'Quasi Federalist' Programme of Decentralisation in Italy since the 1990s: A Side-effect Approach. Local Government Studies, 37(5), 495-516. doi :10.1080/03003930.2011.604543

Mingione, E., Borlini, B., \& Vitale, T. (2009). Immigrés à Milan : faible ségrégation mais fortes tensions. Urbanisme, 362, 83-86.

Tarrow, S. G., Katzenstein, P. J., \& Graziano, L. (1978). Territorial politics in industrial nations. New York : Praeger.

Tosi, S., \& Vitale, T. (2011). Piccolo Nord. Scelte pubbliche e interessi privati nell'Alto Milanese. Milano : Bruno Mondadori.

Vicari Haddock, S. (2005). La rigenerazione urbana: frammentazioni e integrazioni. Dans Le nuove politiche sociali. Problemi dell'azione pubblica. Roma : Carocci. 